\title{
Carnets
}

Revue électronique d'études françaises de l'APEF

Deuxième série - 11 | 2017

Les écrivains écrivent l'Europe en français

\section{La trilogie européenne de Camille de Toledo. Une proposition du « vertige " pour le $\mathrm{XXI}^{\mathrm{e}}$ siècle}

\section{Ana Paula Coutinho}

\section{(2) OpenEdition}

Journals

\section{Édition électronique}

URL : http://journals.openedition.org/carnets/2319

DOI : 10.4000/carnets.2319

ISSN : 1646-7698

Éditeur

APEF

\section{Référence électronique}

Ana Paula Coutinho, « La trilogie européenne de Camille de Toledo. Une proposition du « vertige » pour le XX|e siècle », Carnets [En ligne], Deuxième série - 11 | 2017, mis en ligne le 30 novembre 2017, consulté le 21 avril 2019. URL : http://journals.openedition.org/carnets/2319; DOI : 10.4000/ carnets.2319

Ce document a été généré automatiquement le 21 avril 2019.

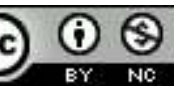

Carnets est mis à disposition selon les termes de la licence Creative Commons - Atribution - Pas d'utilisation commerciale 4.0 International. 


\title{
La trilogie européenne de Camille de Toledo. Une proposition du « vertige " pour le XXI ${ }^{\mathrm{e}}$ siècle ${ }^{\mathbf{1}}$
}

\author{
Ana Paula Coutinho
}

Voici ma question : comment pouvons-nous penser un passage de la « hantise » conflictuelle, paralysante, conservatrice du $\mathrm{XX}^{\mathrm{e}}$ siècle au « vertige » du XXI ${ }^{\mathrm{e}}$ siècle (triple vertige qui est celui de l'identité fragmentée, des origines et des lignées artificielles ou bâtardes et de la perception dans la sédimentation des fictions du monde) sans verser dans la nostalgie?

Camille de Toledo

1 Au cours des dernières décennies, une période de transition entre deux siècles et deux millénaires, nous avons vu défiler plusieurs thèses déclinistes dans différents domaines tels que l'Histoire (Fukuyama, 1992 ; Taguieff, 2000), l'Art (Danton, 1996), l'Économie (Colander, 2000), ou la Littérature (Marx, 2005; Maigueneau, 2006; Todorov, 2006). Ne serait-ce qu'implicitement, elles étaient aussi soutenues par nombre d'imaginaires artistiques puisant dans la catastrophe et le désenchantement par rapport au monde et à l'humanité en général. Par ailleurs, au fur et à mesure des changements géopolitiques et culturels et, donc, à la suite de nouveaux équilibres de force dans le monde, la mise en question de l'identité de l'Europe a visiblement pris de l'ampleur, surtout en ce qu'elle dépasse les intérêts économiques et les structures bureaucratiques de ce que l'on nomme depuis 1993 l'Union Européenne (EU).

2 Déjà à la fin des années 90, les éditeurs de l'ouvrage collectif Les écrivains de la conscience européenne (1997) avaient bien conscience qu'il s'agissait de donner la parole aux écrivains au lieu de se laisser envoûter par les seuls discours des économistes, des politiciens ou d'autres agents de l'appareil administratif de plus en plus lourd de l'Europe communautaire. À cet effet, ils en appelaient à un certain nombre d'écrivains européens, 
certes majeurs, mais qui étaient surtout des voix de la première moitié du siècle, honorable exception faite d'auteurs tels que Václav Havel, Jorge Semprún ou Umberto Eco. L'échantillon s'avérait donc assez réduit pour la deuxième moitié, et ce sans doute du fait d'un univers de référence lui aussi plutôt mince. En fait, du moins au départ, les écrivains contemporains semblent en général moins assertifs, (d'aucuns diront moins « engagés »), dans la production d'une pensée créatrice sur l'Europe, voire sur le monde en général. À la fois cause et conséquence, c'est-à-dire, grâce à un cercle vicieux, les écrivains tout comme les intellectuels semblent désavoués (Sand, 2016) : leurs voix / voies seraient de moins en moins présentes dans les différents forums ou sur les plateaux médiatiques, et la littérature aurait donc perdu le rôle central qu'elle avait joué dans la formation de la conscience historique, esthétique et morale ${ }^{5}$.

Inutile de s'attarder ici sur les raisons intra- et extra-littéraires qui firent en sorte qu'une partie significative de la littérature contemporaine, surtout française ou de tradition francophone ou francophile, se soit abstenue de proposer ouvertement une pensée du monde et de l'humanité (cf. Compagnon, 2006). D'autres, par contre, ont déjà bien entrepris cette radiographie de l'histoire et de la théorie critique ${ }^{6}$. En revanche, il importe de chercher des signes de résistance à cette sorte de renoncement à une " portée projective de la littérature " (Viart, 2010). Autrement dit, il faut aussi tenir compte d'autres auteurs qui, pour diverses raisons, ne se sont pas fait entendre, ou des auteurs plus jeunes ou des voix plus récentes qui distribuent les cartes autrement sur la scène littéraire. Camille de Toledo (1976), pseudonyme d'Alexis Mital, est une de ses voix plus récentes qui entame, depuis le début $\mathrm{du} \mathrm{XXI}^{\mathrm{e}}$ siècle, un parcours de réflexion et de création non seulement dans la littérature mais aussi dans les arts plastiques et la vidéo, dénotant une forte unité de sens dont se dégage une idée d'Europe et pour l'Europe.

En 1985, Italo Calvino développait six propositions pour le nouveau millénaire au cours de ses Leçons américaines (Calvino, 1988) ${ }^{7}$. Quelque trente plus tard, Camille Toledo s'est lancé dans la défense d'une autre et complexe proposition, celle du "vertige». Mais ce qui rassemble le plus ces deux écrivains aux langues et générations différentes, c'est leur fort attachement à la littérature malgré les signes avant-coureurs de sa crise, voire de son agonie. Or, pour Calvino, la confiance en la littérature consistait dans le fait de savoir qu'il y a des choses que seule la littérature, avec ses moyens spécifiques, peut nous donner (Calvino, $2002: 12$ ), tandis que, pour Toledo, la littérature, et notamment les romans, sont porteurs d'un «savoir vertigineux ». Pour lui, c'est cet élan, cette non linéarité, cette profusion et ouverture sur l'inconnu qui, au détriment de sa propre fragilité, permet à l'individu de s'ouvrir au désir d'une refondation (Toledo, 2009: 39), voire d'une refondation de l'Europe elle-même.

\section{Le devoir d'oublier le $X X^{\mathrm{e}}$ siècle}

Au fil des différents discours sociaux, on mêle souvent l'idée d'Europe avec la critique sociale et politique suscitée par maintes dérives tant de l'UE que de ce que l'on appelle la globalisation. Il est donc devenu normal de jouer le Requiem de l'Europe et de sa culture. Par contre, Camille Toledo semble avoir fait le choix de penser et d'écrire à rebours de cette autoflagellation européenne, et ce depuis même qu'il a ouvertement critiqué le fameux « Manifeste pour une littérature-monde » publié le 16 mars 2007 dans Le Monde et signé par une liste significative d'écrivains de langue française. Tout de suite après, en 2008, il se lançait dans la polémique avec Visiter le Flurkistan ou les Illusions de la Littérature 
Monde, entamant ainsi un combat de jeune «Don Quichotte» hétérodoxe, contre des "géants " de la littérature de langue française, où il accusait le célèbre manifeste d'imposer une bipolarisation entre «écrivains de chambre » et « voyageurs » ( Toledo, 2008 : 17). D'après lui, il s'agissait là d'une division aussi naïve et simpliste du point de vue esthétique, qu'astucieuse du point de vue politique (idem: 37). Notons que l'objection de Toledo ne découlait pas exactement de la défense et de la "créolisation" et de la "bâtardise" faite par les "voyageurs" signataires de la littérature-monde, mais de leur illusion par rapport aux périphéries du monde, de leurs traversées " en hôtesse de l'air " (idem : 51), ayant tout simplement oublié ou sous-estimé la «bâtardise originelle» de l'Europe même. À cet effet, il en appelait à l'idée de Mitteleuropa explorée par Claudio Magris dans son long roman-essai, Danubio (1986) dont Toledo ne se privait pas d'exalter les virtualités de pensée sur l'Europe, pour en conclure :

Les voyageurs, dans leur manifeste, auraient pu inscrire notre devenir-créole dans la lignée de cette légende, de cette marche vers les sources du Danube. (...) Plutôt que de pointer la clairière, nous aurions alors eu le choix. Face au deuil, à la perte de l'identité, face au déracinement, non pas seulement le lyrisme de l'ailleurs, du métissage, mais aussi le vertige de l'artifice et l'adieu aux origines (idem : 49).

6 Certes, ce n'est pas Magris qui a inventé les potentialités géoculturelles de la Mitteleuropa, une notion qui au début du $\mathrm{XX}^{\mathrm{e}}$ siècle avait commencé par signifier un projet de confédération politique et économique sous la plume du libéral Friedrich Nauman. Au cours des années 80 , des voix dissidentes des régimes soviétiques étaient parvenues à rehausser le sens d'indépendance intellectuelle et de résistance culturelle de cette région - en gros, l'Europe centrale - face aussi bien au dogmatisme de l'Est qu'aux illusions du consumérisme en Occident. Pensons aux écrivains Milan Kundera et György Konrad et à leurs " Un Occident Kidnappé »" et " Der Traum von Mitteleuropa $~^{10}$ respectivement. Peu de temps après, Claudio Magris devait s'inscrire aussi dans cette réhabilitation culturelle de la Mitteleuropa avec son Danubio, une forme créative de géocritique, une pensée faite de mémoire culturelle et d'émotion esthétique autour du fleuve qui traverse toute l'Europe centrale. Pour Camille Toledo, cette perspective s'avérait bien plus intéressante puisqu'elle permettait d'explorer la fusion de la géographie et de l'imagination dont «Furkistan» est le symbole en tant que pays imaginaire de la littérature (on y reconnaîtra aisément une dérivation du Farghestan de Julien Gracq). Dès lors, Toledo défend qu'au lieu de se mettre en marge de l'Europe ou d'en appeler seulement à d'autres destins, les «voyageurs" de la littérature-monde devraient plonger dans «notre Flurkistan ", plein d'ascendances, de registres vulgaires, d'emprunts et d'exceptions. S'ils l'avaient fait - continuait le jeune écrivain - ils auraient effectivement ouvert les portes d'une autre histoire littéraire : « une histoire non plus française, mais hybride, à la fois bâtarde, créole et post-exotique » (Toledo, $2008: 53$ ).

7 À première vue, on pourrait penser qu'il existerait une contradiction dans le raisonnement de cet écrivain alors que, avant tout, il faisait une véhémente critique au fait de tourner le dos à l'Europe au nom d'autres parages plus « exotiques » et ensuite, il en appelait à l'oubli du $\mathrm{XX}^{e}$ siècle comme principe catalyseur de la " trilogie européenne » dont il allait s'occuper après. Mais ce n'est là qu'une fausse contradiction, comme nous allons le voir.

8 Le premier tome de la trilogie ${ }^{11}$, décrit comme «Essai sur la Tristesse Européenne ", ne cache pas son assise géocritique - la ville de Berlin après la chute du Mur -, ni l'inquiétude existentielle, politique et esthétique qui l'imprègne : comment concevoir le 
futur ? C'est-à-dire, comment peut-t-on survivre à un siècle de catastrophes ? Et l'auteur de souligner d'entrée de jeu l'ampleur de son projet :

Le «nous» qui le traverse est difficilement assignable. C'est le «nous» d'une culture hantée par ses fantômes, le signe d'un commun européen difficile à bâtir ; un «nous » flottant ente plusieurs langues, plusieurs récits, par lequel je cherche à ouvrir une brèche, afin que l'expérience du $\mathrm{XX}^{\text {ème }}$ siècle nous serve à inventer l'avenir, non à hanter éternellement le présent (HB $: \mathrm{s} / \mathrm{p})$. Juste à l'occasion du XXe anniversaire de la chute du Mur de Berlin, Camille de Toledo fait retour sur l'endroit physique et symbolique de cette division de l'Europe (et du monde), pour, dans un premier temps, faire revoir les images de la célébration de la chute du Mur et ensuite faire remarquer tout le symbolisme d'un épisode véridique en novembre 1989 lorsque le grand violoncelliste Rostropovitch, exilé de l'URSS vers les États-Unis, est allé exprès à Berlin, où il jouerait en anonyme, assis auprès du Checkpoint Charlie, les Suites de Bach. La foule qu'y passait ne l'a pas reconnu, et certains passants lui ont même jeté des pièces de monnaie comme à un clochard... Avec le temps, cette scène a pris d'autres significations, notamment celle de rendre plus évidente la force symbolique de cet instant où le «maître " " est clochardisé ", " où - conclut Toledo - l'Europe jeta sa menue monnaie pour que le saltimbanque qu'elle a tant de fois assassiné puisse survivre dans l'ordre nouveau du triomphe » $(2009: 20)$.

Touché par la figure double du désenchantement, aussi bien de l'Est que de l'Occident (HB : 29), l'auteur de Le Hêtre et le bouleau développe ce qu'il désigne lui-même une « lecture spirituelle » de la chute du Mur à rebours des lectures morales et politiques de l'époque, mais qui saisit fort bien les relations plus ou moins souterraines entre le politique et l'émotionnel. Comme l'écrivain le souligne instamment, ce n'est ni le regret ni la nostalgie qui le poussent, sa perspective laissant plutôt percevoir un geste de recul, un recul méditatif qui lui /nous permette de tirer une autre leçon $\mathrm{du} \mathrm{XX}^{\mathrm{e}}$ siècle, au-delà du « rituel liturgique européen du Devoir de Mémoire» (HB : 37), propre à une Europe « ivre de sa mémoire, soûle de ses hontes » (HB:55).

11 L'obsession du passé ou de la pensée de continuité est donc, surtout en Europe, une vraie «hontologie $»^{12}$, pour reprendre une terminologie lacanienne. Dans ce contexte, (se) demande Toledo, comment une culture pourra se reconstituer à partir de la convocation permanente du passé ou d'un simulacre de cimetière, comme c'est le cas de l'« Holocaust Denkmal » au centre de Berlin? (HB : 49). Quel futur pour le vieux continent dont l'union s'est légitimée par le désastre, dont la fondation est intrinsèquement liée à sa propre destruction (HB : 82), insiste l'écrivain?

Les références botaniques que Toledo utilise comme titre de l'essai synthétisent son raisonnement autour des forces animiques qui, en général et d'après lui, ont agi en Europe et dans la culture européenne, dans leur rapport avec le passé et avec le futur. À partir de la définition de "hêtre", un des arbres endémiques en Europe - "Arbre aux feuilles caduques et aux essences nobles » - et tout en jouant avec le mot homophone " être ", l'auteur voit dans cet arbre un symbole, la cristallisation de l'état contemporain de ce continent entre deux siècles. Dans sa lecture, le "hêtre » en vient à absorber un autre paysage physique et symbolique, celui du «bouleau », qui est plutôt caractéristique de l'Europe de l'Est, et qui a fonctionné comme témoin silencieux des pleurs et des exterminations qui s'y sont succédés, que ce soit en Pologne, en Ukraine allemande ou en Russie soviétique... 
13 Ainsi, pour que les Européens parviennent à se libérer de la mélancolie et de la tristesse symbolisées par le hêtre et par le bouleau, à renouveler leurs feuilles, c'est-à-dire leurs fables fondatrices, il faudra, suivant cette allégorie botanique de Toledo, aux inavouables accents deleuziens de rhizome, adopter l'identification avec un autre arbre, à savoir, le banian - «arbre diasporique, dont les branches replongent vers le sol pour reprendre racine plus loin, dans une autre langue, une autre terre » ( HB: 68 ). Ce sera, donc, à l'ombre du banian, de son modèle de vertige, et non plus à l'ombre des deux autres arbres et de leurs modèles moraux que cet écrivain conçoit et aspire à un XXI ${ }^{e}$ siècle polyglotte et hybride pour l'Europe et le reste du monde.

Je reviendrai plus tard sur cette utopie de la pluralité linguistique, sur sa fonction fondamentale dans la pensée européenne de cette trilogie, mais d'abord il faut souligner que l'oubli défendu par Camille Toledo ne peut aucunement se confondre avec la censure ou avec l'ignorance tout court. À l'instar de ce qui est arrivé à Friedrich Nietzsche, à la fin $\mathrm{du} \mathrm{XX}^{\mathrm{e}}$ siècle, Toledo s'est rendu compte de la force tout à fait paralysante du «vice hypertrophié » de l'Histoire (Nietzsche, 2000: 500), au cas où ce culte du Passé ne présuppose pas que la mémoire n'est point une positivité restauratrice en elle-même, et que, par contre, elle implique aussi un travail d'oubli, c'est-à-dire une déviation, un saut, une recréation pour reprendre le Passé qui n'est pas non plus une pure essence qu'il faudrait racheter de la nuit des temps pour le relier au présent. À l'auteur, ces questions qui le / nous touchent:

Qui saura dire à l'Europe qu'il ne faut plus craindre d'échapper à la tristesse, au chagrin, à la mélancolie des cimetières ? Qui osera entailler cet ordre du passé dont l'antitotalitarisme a fait un engagement héroïque? Qui osera dire enfin qu'il faut, pour guérir de la hantise, non pas défendre infiniment le Devoir de Mémoire, mais reconnaitre le travail proprement humain de l'oubli ? (HB : 71).

Dès lors le propos de relance de l'Europe (voire du monde), leur futur en somme, exige que l'on mette en doute les seuls avantages de la «fable de la continuité » et présuppose de quitter des pratiques de mémoire qui, au cours des dernières décennies, ont parfois concouru à une fragmentation du commun à force de communautarisme et ethnicité (HB : 146). Or, cet écrivain croit au contraire que ce futur peut être collectivement créé à partir de la force «du vertige", des expériences de clandestinité et de fragilité de l'être, en somme, de la sagesse qui découle des différentes formes de déplacement, plus ou moins volontaires ou forcées (HB : 148).

\section{Une utopie vertigineuse pour l'Europe}

Les trois ouvrages qui nous occupent ici fonctionnent comme une trilogie justement parce qu'ils interagissent entre eux : l'auteur ne se limite pas au diagnostic spéculatif de l'Europe au moment de transition du XXe au XXI ${ }^{e}$ siècles, mais il poursuit cette enquête sous une autre modalité littéraire, en l'occurrence le roman qu'il fait croiser avec la forme lyrique, expérimentant ainsi un discours autre, intrinsèquement hybride. L'écrivain luimême reconnaît que ce processus délibéré de croisement de fiction, d'autobiographie et de réflexion métalittéraire, représente une infléchissement dans son propre parcours intellectuel, puisqu'il aurait pu se limiter à l'écriture académique, à l'instar de son article «Fragments sur l'u-topos européen : une histoire du vertige, de Robert Musil à Bruce Lee » ( OTD : 153). Mais ce n'était pas vraiment cela qui l'intéressait. Il voulait par ailleurs échapper aux parcours standardisés, sensationnalistes, à la rigueur nihilistes d'autres écrivains 
contemporains. Justement à propos du «telos » de l'écriture littéraire, il n'est pas inutile de rappeler un des "chapitres" au milieu du "roman en pòeme » Oublier, trahir puis disparaitre, où l'auteur / narrateur / personnage médite sous forme de confidence et de témoignage à son fils, lui qui le prolonge et qui porte la responsabilité prophétique d'une nouvelle ère :

J'ai été un écrivain malade, Elias. Je fus atteint par une affliction commune : un pli mélancolique procédant d'une érosion de l'espoir, qui m’obligeait à me tenir voûté. Maigre et pâle comme au chevet d'un enfant. Et cet enfant, c'était le temps ou l'histoire, c'était la disparition des vieilles phrases, des tournures passées (OTD : 87).

C'est lui aussi qui admettra par la suite qu'il avait été atteint de la «maladie de l'Europe ", de sa mélancolie parce qu'il avait refusé de suivre la voie aussi bien des «épopées macabres du vingtième siècle » que des jeux citationnels et des mélanges postmodernistes de de références et de plans - «Batman, Superman ou Godzilla à côté de Primo Levo ou Imre Kertész ». « Ravel et Godzilla, Heidegger et Batman, Platon et Super Mario... » (OTD : 90-91) - avec lesquels une certaine élite artistique en Europe avait cherché à imiter l'Amérique ou l'Asie. Dès lors, il déclare que son sentiment d'étrangeté et de malaise par rapport à l'Europe et à sa culture était à l'époque pareil à celui qui serait de retour à son pays et qui ne reconnaîtrait plus sa culture comme la sienne aussi (OTD : 91-92).

Cette dimension autoréflexive de l'écrivain en tant que sujet de pensée créative faisait aussi partie du "deuxième tome » de la trilogie Vies pøtentielles, un roman composé de trois strates textuelles : les microfictions, les commentaires (Exégèße) et un long poème en vers (Genèße), qui n'est pas sans rappeler « Un coup de dés jamais n'abolira le hasard » de Mallarmé, aux accents de chant apocalyptique. Déjà dans le commentaire exégétique de la première "vie ", nous trouvons une réflexion où se confondent narrateur et écrivain, cette galerie de personnages isolés, de gens fêlés, et ce narrateur Abraham, étant une forme assez double ou ambigüe de mise en écart des devoirs de l'Histoire, de la généalogie et de la filiation. Autrement dit, n'y aurait-il pas là, par excès de pudeur ou d'orgueil, un manque, une faille- s'interroge l'écrivain ? Tourner tout simplement le dos au biographique, et par conséquent aussi à l'Histoire en général, n'est-ce pas choisir une impasse, un faux acte d'oubli à partir du moment que l'on serait en train d'ignorer et de taire ces modules de mémoire, au lieu de les intégrer et de les refaire ? Cet auteur / narrateur, ce nouvel Abraham choisira donc de se concentrer sur différentes « vies », des vies qui ne sont ni vraies ni puissantes, mais plutôt potentielles, intégrant en plus l'étrangeté $d u ~^{13}$ qui peut tout aussi bien renvoyer à la lettre des langues scandinaves qu'au symbole de groupe vide dans le langage mathématique. C'est à partir de ces vies que le narrateur / auteur projette tout d'abord l'« Exégèse ", qui n'est vraiment pas une explication du texte antérieur à la vie, mais plutôt une réflexion en amont, où il se laisse interpeler, notamment par des questions sur sa propre vie et son histoire de descendant de juifs. Ensuite vient la "Genèße », un chant d'apothéose en aval, la proclamation du monde des ørphElins, d'un temps apocalyptique qui suppose non seulement le " Recensement des ancêtres », mais aussi un évidemment, la destruction finale du " format de type homme », ouvrant ainsi sur un Posthumanisme (que l'auteur n'arrive pas à nommer comme tel, il faut dire).

19 Un des aspects qui, à mon avis, se révèle plus intéressant dans tout ce processus tient au fait que Camille Toledo s'est éloigné des sentiers battus de l'archéologie biographique et des voyages de postmémoire des descendants des catastrophes du XXe siècle. À noter aussi qu'il ne s'est pas fait prendre par les effets les plus évidents de l'autofiction, et qu'il 
a cherché à intégrer et à faire dialoguer entre eux les différentes strates de l'existence et de la création, cherchant à faire de façon à ce que son texte reste obstiné et résistant à toute lecture linéaire ou totalisante (VP : 28), et que par-là, à travers non la répétition, mais une réplique des fissures et des schismes existentiels, il puisse contribuer à pousser les limites du pensable (VP : 102-103).

Je n'écris pas en fils mas comme un cobaye. Je me remplis des choses qui nous intoxiquent. Je gobe, puis j'écris comme on tousse. Je cherche à saisir, non pas le petit drame de mes morts, mais ce qu'il reste de nous. Et ce que je découvre en me soumettant à cet ordre détestable, c'est que je ne suis non seulement le fils, mais aussi cette femme qi a détour de l'actualité se fait exploser. Le livre que j'écris est comme elle. Comme moi. Comme tout. En morceaux (VP : 184).

Si nous nous concentrons par exemple sur "Genè§e 3 " (VP : 104-108), nous verrons comment cette écriture « en morceaux », fissurée, se disperse sur la page avec des lettres qui ont l'air d'exploser dans l'espace et des syllabes qui se répandent, produit une image d'ensemble suggérant la « fission » nucléaire de l'être. Il s'agit donc d'une sorte de fiction lyrique qui prolonge et accentue la «fissure de l'homme» imposée par les Génies, des forces obscures dominant le monde, et qui étaient déjà (d)énoncées dans "Genèße 1 ». La vision apocalyptique qui traverse Vies pøtentielles affecte en général tout le monde, mais elle surgit fréquemment appuyée sur quelques références concrètes à l'Europe, pas exaltantes du tout, il faut dire : des images de vieillesse, d'abandon (VP : 23), de nostalgie de la vieille Europe (idem: 54 ) jusqu'aux formes vides ou évidées des transmissions de mémoire, associées à la « diaspora d'imposteurs » que le narrateur / auteur satirise, c'està-dire tous ceux qui sont à la recherche des héritages européens confisqués pendant la Seconde Guerre mondiale au nom du prétendu patrimoine juif (idem : 160).

Cette critique d'un certain mode de vie et de revendication du culte de la mémoire en Europe de la fin du $\mathrm{XX}^{\mathrm{e}}$ siècle s'intègre dans le projet européen sous-jacent à la trilogie dans la mesure où elle met en question les sens de mémoire, de transmission et de continuité, en les faisant confronter avec la force du «vertige », l'appel irrésistible du vide ou de l'inconnu. C'est pourquoi il me semble qu'il est possible de lire ces Vies pøtentielles comme une forme nouvelle de récit d'initiation conduisant et le narrateur et le lecteur à la conception du point extrême du vertige, de la perte du corps, de ses « ramifictions » et annexes (idem : 146).

Cette visée de l'auto-effacement sera poursuivie dans le roman suivant, d'ailleurs déjà synthétisée dans le titre Oublier, trahir puis disparaitre. La dernière station de la trilogie, qui comprend, comme il a déjà été noté plus haut, un voyage en train d'un père et de son fils, évoquant une période particulièrement significative entre la fin $\mathrm{du} \mathrm{XX}^{\mathrm{e}}$ et le tout début du XXI ${ }^{\mathrm{e}}$ siècle. Il s'agit plus concrètement d'un trajet entre deux dates-jalons qui se reflètent en image inversée : 9.11 (1989) - le début de la chute du Mur de Berlin signalant la fin de la Guerre Froide et 11.9 (2001) - les attentats du 11 septembre aux USA et la rupture qui ouvre sur une nouvelle ère dans les relations mondiales, en passant par celle qui fut la fissure la plus tragique et récente à l'intérieur de L'Europe : la guerre en exYougoslavie dans les années 90, symboliquement synthétisée par l'image du pont de Mostar, en Bosnie-Herzégovine, présent aussi sous forme de photographie à l'intérieur de l'ouvrage (OTD : 93). Son hybridité discursive, associant récit, poème et essai vont exactement à la rencontre de l'apologie du croisement et du vertige et représentent une forme de testament poétique et politique pour les générations suivantes, non pas pour ce qu'il leur dit mais pour ce qu'il les oblige à dire/faire. Voici un passage du long poèmeroman : 
J'ai laissé parler le vent qui souffle entre les montagnes.

Je n'ai pas essayé de t'expliquer : ni l'Islam,

Ni le Christ, ni la Croix, ni les vieilles haines d'Europe.

Je me suis retenu, car c'est de toi que je veux apprendre.

Ce monde a trop de passé, il lui faut un avenir, Elias.

Et si j'écris maintenant le récit de notre traversée,

Ce n'est pas pour remplir ta vie,

Mais pour vider la mienne (2014 : 73). de la transmission et à trouver une justification pour le récit-même de leur traversée. En plus, c'est le fils qui annonce vraiment ou prélude au futur ${ }^{14}$. Grâce à une filiation inversée, c'est lui qui recrée ou modèle son père du moment qu'il l'oblige à quitter la douleur du passé à la manière de vieux Européen - «Je viens d'un temps, Elias, où cette disparition / et cet émiettement étaient vénérés » (2014 : 108) -, et à faire un tout autre choix - «être de ce monde, plutôt que du passé » (ibidem) - du point de vue aussi bien existentiel que littéraire. Mais à cette infléchissement dû à la condition de paternité, l'auteur / narrateur va ajouter encore une autre mission - la régénération de l'Europe même :

Si ma maladie était celle de ce vieux continent, si mes trahisons se reliaient à toutes les autres, trahison du passé, de la mémoire, trahison de la libération et de l'égalité, il devait en allait de ma guérison.

(...)

L'Europe, Elias ! grâce à toi, allait guérir (2014 : 142).

Ce «Mélancolic-Europa-Tøur » à deux à travers l'Europe se présente donc comme une forme de guérison de mémoires blessées d'autres trajets $\mathrm{du} \mathrm{XX}^{\mathrm{e}}$ siècle, dont quelques-uns forcés et faits aussi en train... Tout au long du voyage, le représentant du futur amène son père, non pas seulement à se libérer du «bagage superflu " à travers l'intervention mythique de la vieille Gavrilo, un avatar de la Baba Yaga des contes russes, mais aussi et surtout à la compréhension d'un langage nouveau, composé d'un mélange de langues aux sonorités slaves et yiddish ${ }^{15}$, qui prélude à une nouvelle ère de l'«entre-langues», un nouveau chant proclamant la métamorphose vers une nouvelle renaissance de l'Europe.

De l'examen critique continu de son propre parcours en tant qu'écrivain et de celui de «l'état de l'art » de la Littérature fait aussi partie la possibilité de l'extinction tout court de la figure de l'écrivain - cette «figure malade, bileuse et introvertie» ( OTD : 154) inventée par l'Europe et qui désormais semblerait frappée d'obsolescence, d'autant plus que les écrivains seraient toujours en retard sur l'Histoire, "arrivent toujours après " déclare le narrateur de Oublier trahir puis disparaître (OTD :80). Il suffirait de penser aux interrogations suscitées par les livres de Toledo pour démentir la radicalité de ce pronostic, ce qui n'empêche pas l'écrivain de s'imposer un programme d'une autre forme d'écriture, «Entre le format ancien de nos vies et leurs reformatages » (OTD : 236).

Le jour viendra où Elias fera aussi un voyage initiatique avec son, ou ses, descendants. Il leur transmettra ce que la mémoire issue du présent fait révéler dans le passé. Dans le texte qui clôt le roman, intitulé «Le conte du père, du fils, et du verre brisé ", les images et de l'eau versée en continu et du verre brisé demandent à être reçues comme des métaphores du travail et de continuité et de rupture de la transmission, tout comme elles font écho aux tensions entre mémoire et oubli, et entre racine et ramification, qui traversent non seulement ce roman comme les deux autres ouvrages. 
27 Le rapport entre la dimension réflexive de la trilogie et son versant de poétique fictionnelle ne peut non plus être dissocié de son apologie pédagogique du «vertige» appliquée concrètement aux questions de langue, littérature et culture en Europe (et dans le monde en général). D’ailleurs, le premier volume, Le Hêtre et le bouleau, terminait déjà avec la présentation d'un projet très précis - «L'utopie linguistique ou la pédagogie du vertige » dont la praxis inspiratrice est la traduction. Cette «langue commune de l'Europe » au dire d'Umberto Eco exige - ajoute Toledo - que les Européens repensent leurs pédagogies, leurs écoles, leurs histoires et leurs comptabilités afin que la traduction puisse effectivement être au centre de toute l'activité sociale et culturelle. Pour cet écrivain, c'est donc la traduction qui pourrait nous aider à tourner la page du $\mathrm{XX}^{\mathrm{e}}$ siècle en ce que celui-ci a signifié d'exacerbation nationale et identitaire, et à faire l'expérience du vertige, du déracinement et du décentrement telle qu'elle est vécue non seulement par les traducteurs, mais aussi, et de façon particulièrement aigüe, par quiconque est mis en marge par les systèmes ou les frontières : l' "étranger ", le "métèque », le «bâtard », le «créole »... C'est justement à ce propos que Toledo se montrait déjà sensible à, et préoccupé par la montée en Europe de trois partis ou castes irréconciliables, à savoir les nationaux nostalgiques, les europhiles intellectuels et une masse énorme de migrants de la dernière heure, jugés comme extra-Européens (HB : 190). Face à ces divisions symboliques, qui viennent s'ajouter à d'autres limites matérielles et frontières physiques qui se sont accentuées ces dernières années, la proposition de Toledo repose sur trois piliers essentiels et fortement liés entre eux: le plurilinguisme, la traduction et la littérature.

Le programme qu'il y proposait pour 30 ans (2010-2040) en vue du dépassement des contraintes de la nation et de la promotion d'une nouvelle forme de communauté pour "les enfants de l'Europe à naître » (HB : 202) comprenait la création d'une Académie européenne de la traduction, d'un fonds européen pour la traduction, des cours de traduction et d'une société de traducteurs, ainsi que la conception d'un manuel d'histoire européenne. Certains de ces objectifs ont déjà connu une certaine évolution, comme c'est le cas de la création de la Société européenne d'Auteurs ${ }^{16}$, une " contre-institution » qui est simultanément un centre de recherche et un réseau d'auteurs visant 1) la promotion des politiques de traduction, 2) l'action et la pensée entre les langues et 3) la construction d'une citoyenneté multilingue. Liée à trois autres programmes: la liste Finnegan; le projet Borges - Translation and Literary Hub, un outil informatique pour traducteurs et un Observatoire de la Traduction, la Société européenne d'Auteurs essaie surtout de bâtir une coopération entre les langues et de créer un support mondial pour la traduction (World Translation Fund).

29 Camille Toledo est effectivement convaincu que la traduction est le garant de la multiplicité d'identités qui habitent l'Europe (et le monde tout entier), et donc elle représente un versant fondamental de son utopie vertigineuse, car plus que répondre à des finalités très concrètes, plus que résister aux hégémonies, la traduction suppose et facilite une praxis de la transmission et du vertige où travaillent la mémoire de l'original/ el et son dépassement ; une praxis qui apprend à vivre aussi bien dans la proximité et la ressemblance, que dans la distance et la différence...

Enfin, et bien que l'objectif principal de cette brève étude était de présenter les piliers de sa "trilogie européenne", il faudra tout de même souligner que la poétique de cet écrivain dépasse souvent le texte écrit, car Camille Toledo, étant aussi artiste plastique et cinéaste, se fait aussi connaître comme un médiateur infatigable au niveau 
interlinguistique et intermédial ${ }^{17}$. Il s'engage aussi dans la pédagogie du savoir vertigineux de la littérature dans des contextes informels, en dehors des académies, comme c'est le cas du cycle de lectures-conférences intitulé « Une histoire du vertige ${ }^{18}$. Mettons que cette ouverture à d'autres scènes ou à d'autres plateformes d'intervention n'est pas seulement, ni fondamentalement le résultat de la condition d'« intérimaires » des écrivains contemporains, et que Toledo signalait dans Vies pøtentielles (Toledo, 2011 : 127), et nous y décèlerons aussi une praxis liée à la recherche d'une poéthique de la condition d'écrivain en Europe du XXIe siècle. Celle-ci passerait de plus en plus par une résistance à l'instauration progressive d'une société anti-intellectuelle, par la quête obstinée, en somme, d'une effective communauté de différences, donc d'un avenir à rebours de la globalisation hégémonique

\section{BIBLIOGRAPHIE}

AAVV (2010). Relatório Cultural. Progresso Europeu - A Europa le - A literatura na Europa. Instituto de Relações Internacionais (ifa)/Fundação Robert Bosch.

CALvino, Italo (1988). Six Memos for the Next Millennium. Cambridge: Harvard University Press [ed. ut. Seis propostas para o próximo milénio, Trad. de José Colaço Barreiros, Lisboa: Teorema, $4^{\mathrm{a}}$ ed, 2002].

COLANDER, D. (2000). «The Dead of Neoclassical Economics», Journal of the History of Economic Thought, Volume 22, Number 2, 2000.

DANTO, Arthur (1996). Après la fin de l'art. Paris: Seuil [éd. orig.: Beyond the Brillo Box, University of California, 1992].

FUKUYAMA, Francis (1992). The End of History and the Last Man. New York: The Free Press.

KONRAD, György (1984). « Der Traum von Mitteleuropa », Wiener Journal, nº 45.

KUNDERA, Milan (1984). « The Tragedy of Middle Europe », The New York Review of Books, avril 1984.

MAINGUENEAU, Dominique (2006). Contre Saint Proust ou la fin de la littérature. Paris : Belin.

MAGRIS, Claudio (1986). Danubio. Milano : Garzanti.

MARX, William (2005). L'adieu à la littérature. Histoire d'une dévalorisation XVIII-XX ${ }^{\text {ème }}$ siècle. Paris : Minuit.

NIETZSCHE, Friedrich (2000). CEuvres. 1. La Naissance de la tragédie. Considérations inactuelles. Paris : Éditions Gallimard.

PINSON, Jean-Claude (1999). À quoi bon la poésie aujourd'hui ? Nantes : Éditions Pleins Feux.

PINSON, Jean-Claude (2008). A Piatigorsk, sur la poésie. Nantes : Éditions Cécile Défaut.

PINSON, Jean-Claude (2013). Poéthique : une autothéorie. Seyssel : Champ Vallon.

SAND, Shlomo (2016). La fin de l'intellectuel français? De Zola à Houellebecq. Paris : La Découverte 
VIART, Dominique / VERCIER, Bruno (2005). La Littérature française au présent : héritage et mutations de la modernité. Paris : Bordas.

VIART, Dominique (2010). Quel Projet pour la Littérature Contemporaine? Publie.net [première mise en ligne : octobre 2010. Mise à jour : janvier 2017]

TAGUIEFF, André (2000). L'Effacement de l'avenir. Galilée : 2000.

TODOROV, Tzevtan (2006). La littérature en péril. Paris : Flammarion.

TOLEDO, Camille (2008). Visiter le Flurkistan ou les illusions de la littérature monde. Paris : Presses

Universitaires de France.

TOLEDO, Camille de (2009). Le Hêtre et le bouleau. Essai sur la Tristesse Européenne. Paris : Éditions du Seuil.

TOLEDO, Camille de (2011). Vies pØtentielle. Paris : Éditions du Seuil.

TOLEDO, Camille (2014). Oublier, trahir puis disparaître. Paris : Éditions du Seuil.

\section{NOTES}

1. Cet article s'insère dans la recherche menée au sein du Programme Stratégique intégré UID/ ELT/00500/2013 | POCI-01-0145-FEDER-007339 - «Littérature et frontières de la connaissance : politiques d'inclusion ».

2. Les citations seront désormais identifiées par le sigle HB, suivi du numéro de page.

3. Les citations seront désormais identifiées par le sigle VO, suivi du numéro de page.

4. Les citations seront désormais identifiées par le sigle OTD, suivi du numéro de page.

5. Certes, on compte déjà sur plusieurs initiatives, politiques, académiques, éditoriales et autres, autour de la «Littérature européenne». A titre d'exemple, je citerai le Rapport Culturel - Progrès Européen (AAVV,2010), sous le signe de la Littérature en Europe, une édition de L'Institut des Relations Internationales et de la Fondation Robert Bosch, en coopération avec le British Council et les Fondations Pro-Helvetia, Coopération Germano-Polonaise et Calouste Gulbenkian. Au lieu de se concentrer uniquement sur la voix des écrivains, il réunit des témoignages et des réflexions de différents intervenants dans le « champ littéraire » (Bourdieu) » ou dans « discours littéraire » (Maingueneau) qui s'avèrent très pertinentes.

6. Voir, par exemple, les travaux de Dominique Viart, autour du roman surtout (Viart, 2005, 2010 ) et de Jean-Claude Pinson autour de la poésie $(1999 ; 2008 ; 2013)$

7. À savoir: la légèreté, la rapidité, l'exactitude, la visibilité, la multiplicité et la cohérence, quoique l'écrivain ne soit pas parvenu à rédiger cette dernière leçon.

8. Camille Toledo, signant encore à l'époque Alexis Mital, a fondé en 1996 une revue ayant comme titre ce célèbre personnage de Miguel Cervantès, qui interrogeait le monde de cette fin de siècle, marquée aussi bien par la récente de la chute du Mur que par les signes galopants de la globalisation.

9. Publié d'abord en langue anglaise sous le titre The Tragedy of Middle Europe, in The New York Review of Books, avril 1984.

10. Publié dans Wiener Journal, no 45, 1984.

11. Si l'on tient en compte l'ordre de publication, puisque les trois livres n'incluent aucune numération.

12. cf. Leçon du 17 juin 1970.

13. Aussi dans Ørphelins (avec plusieurs variantes dans les majuscules), le nom que Toledo attribue en général à ceux qui portent le « monde de l'après ». 
14. Il s'appelle du reste Elias, comme le prophète de la Bible, reconnu par les trois religions du Livre.

15. Cette ombre du yiddish ne devrait pas être comprise comme acte de respect de la tradition, mais plutôt comme signe d'un élan de pluralité consubstantiel à l'Europe, puisque cette langue de la diaspora juive était apprise toujours en parallèle avec les autres langues.

16. $c f$. http://www.seua.org/fr/

17. Voir à propos de sa condition d'artiste amphibien, dans la tradition d'autres artiste comme, par exemple, Margeurite ou Chris Marker, Audrey Evrad, «On Impossible Cinema : Camille de Toledo's Intermedial Archaeology of the Fictions of Our Times ", Revue critique de fixxion française contemporaine, 2012, accessible sur http://www.revue-critique-de-fixxion-francaisecontemporaine.org/rcffc/article/view/fx07.10/784

18. Ce cycle a eu lieu de septembre 2016 à juin 2017 à la Maison de la Poésie de Paris en partenariat avec Diakritic et remue.net https://diacritik.com/2016/10/17/une-histoire-duvertige-camille-de-toledo

\section{RÉSUMÉS}

C'est dans le contexte actuel de visions catastrophiques ou de raréfaction mélancolique d'écritures propositionnelles sur l'Europe, sur le monde et, en général sur l'avenir de l'humanité, mais simultanément à un moment d'infléchissement éthique dans la littérature (et de narrativisation de l'éthique), qu'il semble intéressant, voire important, d'analyser la "trilogie européenne " d'un jeune écrivain comme Camille de Toledo, constituée par un essai et deux romans - Le Hêtre et le bouleau. Essai sur la Tristesse Européenne $(2009)^{2}$, Vies pøtentielles $(2011)^{3}$ et Oublier, trahir puis disparaître (2014) ${ }^{4}$. Je me pencherai surtout sur sa façon de mettre en relation la mémoire et l'oubli, en tant que processus de dépassement des blocages existentiels et de création hérités $\mathrm{du} \mathrm{XX}^{\mathrm{e}}$ siècle, ainsi que sur sa proposition du "vertige » en tant que métaphore de (re)création utopique.

Considering the current context of catastrophic visions and melancholic scarcity of propositional writings on Europe, on the world and, in general, on the future of humanity, but also taking into account the «ethical turn» in literature (and the narrativisation of ethics), it seems interesting, and even important, to analyze the "European trilogy" of a young writer such as Camille de Toledo, which includes an essay and two novels - Le Hêtre et le bouleau. Essai sur la Tristesse Européenne (2009), Vies pØtentielles (2011) and Oublier, trahir puis disparaître (2014). I will focus on how the author relates memory and oblivion, as a process of overcoming existential and creative blockages inherited from twentieth century's legacy, as well as on his proposition of "vertigo" taken as a metaphor for a (re)utopian creation.

\section{INDEX}

Mots-clés : Camille de Toledo memory and forgetting, Europe, vertigo, translation Keywords : Camille de Toledo, mémoire et oubli, Europe, vertige, traduction 
AUTEUR

ANA PAULA COUTINHO

Un. Porto - ILCML - APEF

amendes@letras.up.pt 